FORMATION Formation emploi

Revue française de sciences sociales

137 | Janvier-Mars 2017

La formation continue en contexte : l'entreprise au cœur des enjeux

\title{
Comment les entreprises s'organisent pour mettre les salariés en capacité de se former
}

How do firms organized in order to enhance the capability for learning of their employees

Wie organisieren sich Unternehmen, um bei Arbeitnehmern die

Fortbildungskapazität zu fördern?

Cómo se organizan las empresas para darle a los asalariados capacidad de

formarse

Josiane Vero et Jean-Claude Sigot

\section{CpenEdition}

\section{Journals}

Édition électronique

URL : http://journals.openedition.org/formationemploi/5011

DOI : 10.4000/formationemploi.5011

ISSN : 2107-0946

Éditeur

La Documentation française

Édition imprimée

Date de publication : 30 avril 2017

Pagination : 73-75

ISSN : 0759-6340

Référence électronique

Josiane Vero et Jean-Claude Sigot, «Comment les entreprises s'organisent pour mettre les salariés en capacité de se former », Formation emploi [En ligne], 137 | Janvier-Mars 2017, mis en ligne le 30 avril 2017, consulté le 30 octobre 2020. URL : http://journals.openedition.org/formationemploi/5011 ; DOI : https://doi.org/10.4000/formationemploi.5011 


\title{
Comment les entreprises s'organisent pour mettre les salariés en capacité de se former
}

\author{
Josiane Vero \\ Economiste, chargée d'études au Céreq dans le département Formation et Certification \\ Jean-Claude Sigot \\ Economiste et statisticien, chargé d'études au Céreq dans le département Formation et \\ Certification
}

Résumé

\begin{abstract}
Comment les entreprises s'organisent pour mettre les salariés en capacité de se former

Depuis près de quinze ans, les réformes de la formation continue, en France, visent de plus en plus à faire du salarié le principal acteur de sa formation et de son évolution professionnelle. Une nouvelle sémantique du travail s'installe, associant la liberté à la responsabilité. Cette contribution explore les conditions de ce couplage entre liberté et responsabilité en matière de formation continue. À partir de l'approche par la capacité d'A. Sen, elle évalue la portée des politiques de formation des organisations pour identifier celles qui favorisent la capacité des salariés à agir sur leur formation. Elle propose une typologie de ces politiques de formation et identifie les caractéristiques des organisations les plus capacitantes, à partir de l'enquête couplée employeur-salarié DIFES 2 (dispositif d'information sur la formation employeur-salarié).
\end{abstract}

Mots clés : formation professionnelle continue, politique de la FPC, FPC en entreprise, approche par les capabilités, cheminement professionnel

Abstract

How do firms organized in order to enhance the capability for learning of their employees

For nearly 15 years, reforms in continuing vocational training implemented at the French policy-making level have increasingly expected the employee to become the main actor of his learning and his professional evolution. A new semantics of work and learning is emerging, combining freedom and responsibility. This contribution explores the conditions of this linkage between freedom and responsibility with regard to continuing vocational training. Drawing inspiration from the capability approach of Amartya Sen, it assesses the scope of corporate continuing vocational training policies to identify those that promote the capability for training of employees. It provides a typology of these corporate trai- 
ning policies and identifies the characteristics of the most capability-friendly organizations from the French linked employer-employee survey DIFES2.

Keywords: continuing vocational training, CVT policy, CVT in companies, capabilities approach, occupational paths

Journal of Economic Literature: J 24 ; M 51

Traduction : Auteurs

C'est peu dire que depuis une bonne quinzaine d'années, les politiques de formation continue sont traversées par un courant de réformes ininterrompues qui déplacent progressivement "le centre de gravité du droit de la formation du collectif vers l'individuel " (Luttringer, 2013).

Avec l'Accord national interprofessionnel (ANI) du 14 décembre 2013 et la loi 5 mars 2014 relative à la formation professionnelle, à l'emploi et à la démocratie sociale s'engage la dernière réforme en date. Si elle prévoit des transformations impactant tous les acteurs de la formation continue, c'est peut-être et surtout l'individu qui est placé au cœur du système. Il est, plus que jamais, appelé à être "l'acteur de son évolution professionnelle » (ANI 14 déc. 2013, article 1 du titre I).

Le dispositif le plus important pour atteindre cet objectif semble être le compte personnel de formation $(\mathrm{CPF})$. Il consacre un droit attaché à la personne, au-delà de son contrat de travail, qui a pour objet de "donner à chacun les moyens de sécuriser son parcours professionnel» (ANI 14 déc. 2013, préambule du titre III). Cette tendance à l'individualisation des droits ne s'est pas démentie avec la loi El Khomri du 8 août 2016. Avec sa mesure phare, le compte personnel d'activité (CPA), elle consolide les murs porteurs d'une protection que les actifs ne trouvent plus dans le contrat de travail (Dayan, 2016). Ils sont désormais appelés à prendre en charge leur sécurité en mobilisant un ensemble de "comptes personnels ayant pour objet direct ou indirect la formation" (Luttringer, 2016) face aux aléas de la vie professionnelle.

On relève pour la première fois l'énonciation d'un objectif de ce type dans le préambule commun aux ANI de 2003 et 2009 : "Chaque salarié doit pouvoir être acteur de son évolution professionnelle ». Étroitement associée au défunt Droit Individuel à la Formation (DIF), qui sera remplacé par le CPF, cette injonction lancée alors aux seuls salariés s'est accompagnée de l'instauration du thème de la responsabilité individuelle.

Rompant avec le système issu de la loi de 1971 qui privilégiait, en premier lieu, l'entreprise, ses financements et sa responsabilité, les partenaires sociaux et les pouvoirs publics se sont ainsi engagés à adapter la formation continue aux transformations de l'emploi et du travail, en instituant un droit individuel à la formation qui favoriserait le développement des formations à l'initiative de l'individu (Merle et Lichtenberger, 2001). En 
positionnant ainsi l'individu au centre, ce déplacement de responsabilité participe d'un double mouvement qui, d'une part, valorise la liberté individuelle et, d'autre part, exige que chacun prenne ses responsabilités et soit le responsable de sa formation et de son évolution professionnelle.

La valorisation de la responsabilité individuelle et de la liberté est l'un des traits essentiels de la sécurisation des parcours professionnels, modalité française de la flexisécurité européenne (Commission européenne, 2007). Elle est apparue dans un contexte de fragilisation du modèle fordiste d'emploi et de protection sociale qui a pourtant formé l'ossature d'une régulation sécurisée au travers de laquelle les travailleurs échangeaient subordination contre sécurité dans le cadre d'emplois à temps plein et à vie dans la même entreprise. À cette dominance des marchés internes du travail associée à une "sécurité de l'emploi " (Boyer et Durand, 1993) se substitue progressivement une problématique de sécurisation active des parcours (Giddens, 1998), où le travailleur, perçu comme plus mobile, est appelé à prendre en charge des transitions (Schmid et Gazier, 2002) entre différents statuts dans l'emploi, le travail et la vie active en général (Supiot, 1999). En contrepartie, il revient aux politiques publiques de l'équiper à cette fin.

Or, déplacer la responsabilité de la formation de l'entreprise vers le salarié constitue une évolution ambivalente. Le pouvoir d'agir de l'individu est encouragé, mais dans le même temps, celui-ci est tenu pour seul responsable d'un éventuel déficit de formation, d'une absence d'évolution professionnelle ou d'un manque "d'appétence ", indépendamment des contraintes structurelles qui pèsent sur lui. La responsabilisation de la personne dépend autant des droits qui lui sont attribués que des conditions de leur effectivité, faisant de la conversion des droits formels en droits réels dotés d'effets un enjeu central. Aussi, se soucier de la liberté d'action suppose-t-il d'analyser le droit de la formation, mais aussi de sortir du droit pour s'aventurer sur le terrain de sa mise en œuvre, plus particulièrement l'entreprise, principal canal de formation des salariés, en France (Caillaud et Zimmermann, 2011). Il importe donc de se doter d'instruments susceptibles d'évaluer la portée des politiques de formation mises en œuvre dans les organisations pour apprécier celles qui favorisent la capacité d'action des salariés en matière de formation et de parcours professionnel (Verd et Vero, 2011).

Notre contribution vise à proposer un tel instrument analytique, inspiré de l'approche par les capacités d'Amartya Sen $(2000 ; 2005 ; 2006)$. Nous présenterons d'abord les concepts clefs de l'approche, avant d'examiner en quoi et comment elle offre un cadre analytique pertinent pour apprécier la capacité des personnes en matière de formation. Nous analyserons ensuite quatre configurations d'entreprise, selon qu'elles favorisent plus ou moins cette capacité, à partir de l'enquête couplée employeur-salarié DIFES 2 (dispositif d'information sur la formation employeur-salarié, encadré $\mathbf{1}$ ). La dernière partie interrogera les caractéristiques structurelles des organisations les plus propices à la capacité à se former. 


\section{Analyser les politiques de formation à l'aune des capacités}

Une abondante littérature existe, qui tente de comprendre la réalité organisationnelle des entreprises, en essayant de repérer des formes récurrentes (Mintzberg, 1982 ; Nizet et Pichault, 2001 ; Lorenz et Valeyre, 2000 ; Livian, 2008). Ce faisant, elle interroge les relations entre les transformations organisationnelles et les politiques de formation. Ce désir de mettre en ordre se fonde sur "une tradition d'intelligibilité par classification " (CoenenHuther, 2007).

Passer la formation continue en entreprise au filtre de l'approche par les capacités n'est pas neutre. Comme pour toute classification, la base informationnelle sur laquelle elle repose véhicule des présupposés proposant un reflet particulier des politiques de formation. Elle en fournit une image partielle en sélectionnant des informations, aux dépens d'autres possibles (Vero et al., 2012). De la sorte, elle contribue aussi à reconfigurer la situation observée dans le sens des valeurs et normes qui lui sont sous-jacentes. Dans notre cadre, quelles sont la focale et l'échelle d'une approche en termes de capacité ?

\subsection{La focale de l'approche par les capacités}

L'approche par les capacités, proposée par Amartya Sen, constitue une contribution majeure aux théories de la justice. Elle focalise son attention sur "la liberté réelle de chacun de conduire la vie quil a des raisons de valoriser". Elle développe une conception exigeante de la liberté, fondée sur la participation démocratique, l'accès aux opportunités et le pouvoir d'agir. Les capacités visent à donner un contenu réel, au-delà de ses aspects formels, à l'idée de liberté. Une des spécificités de l'approche est de se situer à la charnière entre une perspective normative, qui fait de l'égale distribution de cette liberté un principe de justice (Sen, 2009), et une perspective descriptive d'évaluation de la liberté d'agir. Dans une perspective normative, Sen plaide pour une conception renouvelée de la justice, fondée sur l'égale distribution de la liberté réelle que l'action publique doit chercher à étendre. Dans une perspective descriptive, l'approche par les capacités invite à "faire surgir des faits utilisables " (Farvaque, 2008) à partir d'une problématisation de la liberté selon plusieurs dimensions susceptibles d'être appréhendées à travers une enquête empirique. Nous nous appuierons sur ces quatre dimensions fondamentales (Zimmermann 2014 ; Subramanian et Zimmermann, 2013 ; Lambert et Vero, 2013).

La première dimension renvoie à l'idée d'objectifs qui se focalise sur la pluralité de fins et d'objectifs que peuvent poursuivre les êtres humains. Les buts et les projets sont divers d'une personne à l'autre, selon leurs aspirations, leur origine sociale, leur conception du travail. Cet accent mis sur la diversité des objectifs soulève la délicate question des préférences adaptatives, à savoir qu'une personne qui vit depuis des années dans une situation de choix restreint peut se résigner à cette situation; elle n'est alors plus en mesure de se 
projeter dans d'autres espaces de possibles que celui de son quotidien, aussi restreint futil. Sen (1985) suit ainsi Jon Elster (1983) en confirmant que "notre lecture de ce qui est faisable dans notre situation et notre position peut être cruciale sur les intensités de nos désirs, et peut même affecter ce que nous osons désirer». Ce phénomène de préférences adaptatives en matière de formation a été mis en évidence par Lambert et Vero (2013). Paradoxalement, plus une entreprise développe une politique de formation ambitieuse, plus augmente la probabilité que les salariés déclarent des besoins de formation non satisfaits, alors qu'inversement, moins l'entreprise est formatrice et ouvre des espaces de débat autour de la formation, moins les salariés déclarent de besoins insatisfaits et moins ils aspirent à se former. L'absence de liberté réelle d'accomplir inhibe jusqu'à la liberté de se projeter. Pour autant, la définition des objectifs auxquels la personne accorde de la valeur ne doit pas être confisquée par l'entreprise ou des experts. Elle fonde la nécessité, pour chacun, de construire des objectifs dans un débat informé. Comment analyser cette dimension dans le domaine de la formation ? Elle peut être abordée sous forme de questions au regard de la diversité des objectifs que l'entreprise assigne à la formation : à quel type de formation les salariés peuvent-ils prétendre - une formation qui réponde à leurs attentes ou exclusivement à celles de l'entreprise ? - et que peuvent-ils espérer de la formation - une simple adaptation au poste, une évolution professionnelle ou une autre réalisation de valeur ?

La deuxième dimension concerne la liberté-opportunité qui pose la question de l'étendue et de la qualité des opportunités accessibles à une personne dans une situation. Elle dépend autant de l'accès aux ressources que de la possibilité de les convertir. Ainsi le DIF, le CPF ou l'entretien professionnel obligatoire sont autant de ressources mobilisables pour un salarié, mais au-delà de ces ressources disponibles, il faut considérer ce que les personnes en font au regard de leurs possibilités réelles. C'est là un des principaux apports de l'approche. Divers facteurs individuels, sociaux ou environnementaux, agissent comme un facteur d'obstruction : faible investissement en formation de l'entreprise, manque d'information, refus de l'entreprise d'organiser des entretiens pour toutes les catégories de salariés, désaccord avec le supérieur hiérarchique sur la formation, contraintes de conciliation entre vie professionnelle et vie familiale, etc ${ }^{1}$.

Ainsi dans le domaine de la formation, il convient de s'interroger sur les types de formations qui sont accessibles (leur durée, leur variété, leur coût), à qui (l'accès à la formation est-il réservé à certaines catégories de salariés ou l'entreprise offre-t-elle une égale répartition des opportunités) et avec quelle latitude de choix.

Les opportunités de formations entretiennent un lien étroit avec l'organisation du travail. Il peut paraître difficile, selon les cas, d'établir une ligne de partage claire entre l'exercice du travail et la formation (Pichault et Nizet, 2013). Cette perméabilité de la formation et de l'activité professionnelle rend alors difficile la mesure des opportunités réelles

1. De ce point de vue, le concept de capacité résulte d'une articulation entre l'individuel et le social (De Munck et Zimmermann, 2008). 
à partir d'indicateurs tels que le nombre moyens d'heures de formation par personne ou le volume de la masse salariale consacré à la formation. Aujourd'hui, les entreprises déploient de nouvelles formes d'organisation du travail et de mobilisation des salariés qui engendrent la disponibilité et l'implication fondée sur l'initiative, l'autonomie et la responsabilité. L'organisation du travail connaît par conséquent des transformations susceptibles de favoriser des opportunités d'apprendre là où les cahiers des charges tayloriens ou bureaucratiques limitaient ces possibilités. Pour appréhender ces opportunités de formation informelle, notre parti pris est de chercher à prendre en compte, au-delà des actions de formations planifiées, les opportunités qu'autorise l'organisation du travail. En matière d'opportunités, il conviendra donc d'apprécier si les salariés sont réduits au rôle de simples exécutants ou si l'organisation du travail comporte une dimension cognitive et d'apprentissage. Il s'agira aussi de se demander si ces modes d'organisation plus apprenants sont susceptibles de développer la capacité des salariés à agir sur leur parcours.

La troisième dimension, la liberté-processus, renvoie aux processus qui conditionnent "l'exercice d'un libre choix dans l'action" (Sen, 2000, p. 32). Au-delà des ressources et des opportunités, des processus autorisent les personnes à déterminer ce qui fait sens pour elles, mais aussi à s'exprimer et faire entendre ce qui est de valeur dans une situation donnée (Bonvin, 2008). Exprimer son point de vue et le faire entendre a trois formes de légitimité chez Sen (1999) : une légitimité intrinsèque car il y a amélioration de la qualité de vie, quelle que soit l'issue du débat; une légitimité instrumentale qui donne plus de résonance aux revendications des personnes et permet leur meilleure prise en compte dans les processus de décision collective. Cette fonction instrumentale peut passer par la personne ou être médiatisée par des acteurs collectifs, tels que les syndicats, représentant les intérêts des salariés et leur donnant un poids dans le débat. Enfin, une légitimité constructive car la démocratie "permet aux citoyens d'apprendre les uns des autres" (Bonvin, 2005, p. 25). La formation des aspirations n'est donc pas une affaire d'individu mais de délibération collective. Les préférences, aspirations, désirs et besoins ne sont pas donnés indépendamment de la délibération, mais élaborés et révisés au cours de celle-ci. Ainsi, en matière de formation continue, l'entretien professionnel peut soutenir la liberté-processus, à condition qu'existe une véritable liberté d'expression. En matière de liberté-processus, il importe de déterminer quels sont les acteurs, les pratiques, les procédures impliqués dans l'accès aux opportunités, mais aussi dans le choix d'une formation en particulier. Dans quelle mesure les salariés peuvent-ils faire entendre leur voix et leurs souhaits, influencer les décisions en matière de formation et d'évolution professionnelle? Quel rôle jouent les acteurs collectifs ?

Rendre compte de la liberté-processus suppose aussi de lier la politique de formation aux formes de management. La formation peut être l'instrument d'une politique de mobilisation de la main-d'œuvre qui s'appuie sur l'implication contrainte (Durand, 2004) ou l'autonomie contrôlée (Appay, 2005). L'autonomie accordée aux salariés ne serait 
qu'une manière de garantir la subordination (de Gaulejac, 2005 ; 2015), de mobiliser le meilleur de chacun au service de la performance de l'entreprise (Giauque, 2003), aboutissant ainsi à un contrôle généralisé des travailleurs. Selon cette vision, l'âge de l'autonomie ne serait que poudre aux yeux, un vernis visant à donner une image de respectabilité à des pratiques qui resteraient tout autant hiérarchiques et contraignantes. En matière de liberté- processus, il convient de se demander, par exemple, si la fixation d'objectifs favorise des modes participatifs et des marges de manœuvre permettant aux salariés d'avoir une prise sur leur devenir. Les nouvelles formes organisationnelles accordant plus de place à l'autonomie et à l'initiative sont-elles une manière de mobiliser le meilleur de chacun au service d'objectifs définis par la hiérarchie ? Ou laissentelles une plus grande place à l'initiative de leurs membres?

Enfin, dans la perspective des capacités, l'idée de réalisation de valeur est érigée comme une autre caractéristique essentielle. Amartya Sen s'est attaché à pointer les limites d'une conception ressourciste de l'égalité pour penser les libertés réelles des individus. Le problème, selon lui, réside dans ce que cette approche se centre sur les droits formels ou les ressources de la liberté, et non sur son étendue, c'est-à-dire sur ce que les individus peuvent obtenir à partir de ces ressources. Autrement dit, ce qui fait défaut dans cette approche ressourciste, c'est une information sur la manière dont les individus peuvent réaliser des buts et des projets auxquels ils accordent de la valeur. Le degré de réalisation des buts et projets constitue, en bref, les résultats (outputs) des actions. Dans l'esprit de Sen, à quoi bon des droits à la formation si la personne n'est pas capable de s'en servir ? Cette idée de réalisation de valeur est centrale pour comprendre le concept de capacité, mais on ne peut appréhender son pouvoir heuristique sans y adjoindre les autres registres complémentaires que sont les objectifs, les opportunités et les processus. Dans notre cadre, cette dimension peut être déclinée sous forme de questions : quel type de formation les salariés réalisent-ils - une formation qui réponde à leurs attentes ou exclusivement à celles de l'entreprise ? - et que peuvent-ils espérer de la formation une fois réalisée - une simple adaptation aux conditions de travail ou une contribution à un projet professionnel qui a de la valeur à leurs yeux ?

Nous retiendrons ces quatre dimensions - objectifs, opportunités, processus, et réalisations de valeur - comme éléments d'analyse de la capacité des salariés à se former.

\subsection{L'échelle d'analyse de l'approche par les capacités}

Les théoriciens de l'organisation ont élaboré de nombreuses typologies au niveau de l'individu et de son espace de travail - microstructures - ou sur l'organisation au niveau global - macrostructure (Livian, 2008).

Parmi les premières, nous relèverons celle de Lorenz et Valeyre (2005) qui met en évidence que les nouvelles formes d'organisation du travail dans l'Union européenne ne relèvent pas du seul modèle de la lean production (Womack et al., 1990), où les 
salariés disposent d'une autonomie limitée et contrôlée sous des contraintes de rythme importantes. Ils mettent en évidence aussi celui des organisations apprenantes (Senge, 1990), où les salariés bénéficient d'une large autonomie et apprennent continuellement ensemble. Cette classification révèle la pluralité des modèles d'organisation alternatifs aux organisations tayloriennes. Mais plutôt que l'organisation, c'est l'environnement des salariés au travail que les auteurs caractérisent. En tenant à l'écart l'organisation comme entité, cette approche conduit à une certaine myopie. Certes, des déterminants organisationnels ont été pris en considération, à travers la thématique des conditions et des relations dans le travail, mais de façon limitative.

Mais quelle plus-value peut-il y avoir à changer d'échelle pour partir à la recherche de l'organisation capacitante ? Et quelles sont les dimensions qu'il conviendrait d'adjoindre pour l'analyse ? Afin de répondre à ces questions, un détour par la notion d'organisation s'impose (Livian, 2008). Alors que la notion d'environnement se focalise sur les éléments qui affectent l'activité dans une situation, la notion d'organisation introduit des dimensions qui ne relèvent pas forcément de l'environnement immédiat de travail. Elle mobilise une conception variable de l'espace, faite aussi bien de proximité que de distance par rapport à l'activité. En outre, elle engage aussi bien les orientations des politiques des employeurs, le pouvoir d'action des acteurs collectifs que les réalisations de valeur des salariés.

La question qui se pose ensuite est celle de savoir quel dispositif d'enquête peut rendre compte de cette intrication entre structure organisationnelle, environnement immédiat de travail et liberté réelle des personnes, qui ne doivent pas être considérées comme de simples instruments au service d'objectifs de compétitivité et de performance. De ce point de vue, l'approche par les capacités laisse la question de la méthode ouverte, invitant le chercheur à définir une méthodologie adaptée. Elle appelle néanmoins une approche multi-niveaux (Salais, 2007 ; Corteel et Zimmermann, 2007 ; Lambert et Vero ; Lambert et al., 2012). La réflexion que nous avons menée sur son opérationnalisation, sur la façon dont des possibles, des espaces d'opportunités et des choix personnels peuvent être saisis et analysés, nous a amenés à adopter une méthode quantitative articulant des interrogations au niveau des salariés, mais aussi au niveau des représentants de l'employeur. C'est donc à partir du "dessous " et du " dessus " que l'on peut chercher à observer la capacité d'agir en matière de formation. À cet égard, l'enquête couplée employeur-salarié DIFES2 (cf. encadré 1) saisit l'organisation au sens de la définition de l'entreprise par l'INSEE, soit «la plus petite combinaison d'unités légales qui constitue une unité organisationnelle de production de biens et de services jouissant d'une certaine autonomie de décision, notamment pour l'affectation de ses ressources courantes $»^{2}$.

2. https://www.insee.fr/fr/metadonnees/definition/c1496, consulté le 9 février 2017. 


\section{Encadré 1 : Les enquêtes}

L'enquête CVTS4 : Il s'agit de la vague 2010 de l'enquête Continuing Vocational Training Survey, coordonnée par Eurostat. En France, elle est conduite par le Céreq, en collaboration avec la Dares (Direction de l'animation de la recherche, des études et des statistiques) et l'Insee, auprès de 5360 entreprises de 10 salariés et plus du secteur privé et semi-public, hors agriculture, éducation et santé. Elle porte sur la formation continue pendant les heures de travail ou financée, au moins en partie, par l'entreprise pour son personnel.

Le dispositif DIFES2 ou Dispositif d'information sur la formation employeur-salarié : II est constitué d'un volet employeur et d'un volet salarié. Le volet employeur est une sélection de 1813 entreprises répondantes à CVTS4. Le volet salarié regroupe un échantillon de 6070 salariés présents dans ces 1813 entreprises en 2010 (hors intérim) et suivis jusqu'au premier trimestre 2012, date de l'enquête. Sont recensés et décrits les formations suivies, mais aussi l'organisation du travail, les conditions de dialogue dans l'entreprise et les changements dans le travail.

\section{Quatre configurations contrastées d'entreprises}

Pour mettre en évidence les principales configurations organisationnelles, une classification hiérarchique ascendante (en Annexe de la version électronique du présent article) a été réalisée, à partir du volet entreprise, sur trente-six variables issues de l'enquête CVTS4, relevant de trois registres : sept variables concernent les objectifs de la formation, vingt traduisent les opportunités et neuf les processus. Notons que les réalisations de valeurs ne peuvent être appréciées qu'à partir du volet salarié. Elles ne sont pas constitutives de la classification hiérarchique ascendante, mais elles sont mobilisées comme variables illustratives permettant de décrire plus finement les configurations.

Les objectifs de la formation constituent le premier registre de variables mobilisées pour caractériser les organisations. À la suite de Perrow (1970), Etzioni (1971), Mintzberg (1986) ou Nizet et Pichault (2001), nous considèrerons que les organisations poursuivent généralement plusieurs finalités. Nous distinguons, d'une part, des finalités exclusivement orientées vers l'entreprise (recherche de flexibilité, atteinte de performance, respect d'une norme de qualité, diffusion de valeurs auxquelles les salariés sont censés adhérer) ; d'autre part, des objectifs orientés vers les salariés (développement des salariés, perspectives de mobilité horizontale ou promotionnelle).

Quant aux opportunités de formation, elles sont appréhendées de deux manières. D’une part, elles incluent des possibilités de formation formelle. La caractérisation de ces opportunités repose sur des indicateurs concernant le niveau d'investissement réalisé par les entreprises, comme la masse salariale consacrée à la formation ou l'espérance moyenne des actions mises en ouvre. Les indicateurs incluent aussi la variété et l'incidence des modalités de formation, depuis les formes les plus encadrées que sont les 
cours et stages, jusqu'à l'autoformation, en passant par les formations en situation de travail, la rotation sur poste, les cercles de qualité, voire les séminaires, etc. La manière dont s'opère la répartition plus ou moins inégalitaire des formations entre les divers groupes socio-professionnels est une troisième caractéristique centrale. L'existence d'un plan de formation constitue le dernier indicateur. D'autre part, elles incluent des opportunités de formation informelles, c'est-à-dire caractérisées par des situations de travail qui placent les salariés dans une dynamique d'apprentissage. De ce point de vue, les différentes formes d'organisation du travail sont à prendre en compte, qu'elles favorisent ou contrarient le développement du contenu cognitif du travail, qu'elles développent ou entravent la délégation d'autonomie, qu'elles favorisent ou empêchent un fonctionnement en équipe de travail, qu'elles recherchent ou non la gestion de la qualité, qu'elles déploient ou pas la rotation de tâches, etc. En matière d'opportunités de formation, les formes d'organisation du travail peuvent agir dans un sens tout aussi exclusif que les politiques de formation formelle. Comment ces deux types d'opportunités se combinent-ils ?

Les processus d'accès à la formation sont appréciés au regard de deux questions génériques. Qui peut délibérer et qu'est-ce qui est ouvert à la délibération ? La première interrogation amène à appréhender, d'une part, si les entretiens professionnels sont généralisés ou réservés à certaines catégories ; d'autre part, si les représentants du personnel sont impliqués dans la gestion de la formation. La seconde interrogation conduit à apprécier l'information mise à disposition par l'entreprise (tant sur les possibilités concrètes de formation que sur l'accès au DIF) et l'existence d'un interlocuteur dédié à la formation (une personne ou un service) susceptible de fournir des informations complémentaires. L'information détermine le contenu de ce qui peut être délibéré. Rendre compte simultanément de l'ensemble des dimensions qui définissent les processus suppose de surcroît de lier la politique de formation aux dynamiques managériales et à la manière dont le pouvoir est distribué au sein de l'entreprise. Cela passe par un repérage des modes de management qui peuvent allier la fixation d'objectifs généraux concernant les résultats du travail (individuels et/ou collectifs) et l'évaluation des compétences au déploiement de marges de manœuvre ou, au contraire, être associés à un renforcement des systèmes de contrôle (Bonvin, 2009).

Les réalisations de valeurs concernent la formation décrite à travers ses finalités (pas de finalité, en lien avec un changement dans l'emploi ou le travail, en lien avec un projet professionnel).

La combinaison de ces variables conduit à l'énoncé d'une typologie qui distingue quatre configurations : structure simple, ajustement au poste de travail, développement des compétences et développement des capacités (Sigot et Vero, 2004). 


\section{Structure simple}

L'existence d'opportunités de formation est une condition nécessaire au déploiement des capacités. Or, cette première configuration, qui regroupe $43 \%$ des entreprises et $15 \%$ des salariés, ne remplit pas, ou mal, cette condition. Elle se singularise par son absence de stratégie formelle autour des différentes dimensions que sont l'organisation du travail, la gestion des ressources humaines et la politique de formation. Ces entreprises font d'ailleurs un usage minimal de la formation ; les dépenses correspondent en effet au minimum légal et offrent peu d'opportunités de formation, mais aussi des espaces de discussion peu formalisées et peu participatifs. Cette configuration se définit également par la faible autonomie accordée aux salariés et le faible contenu cognitif du travail. Le travail en équipe, la rotation des tâches et la gestion de la qualité y sont peu diffusés, mais les contraintes de travail y sont moindres aussi. Ce profil présente un certain nombre de caractéristiques relevant des modèles d'organisation en structure simple et définies par Mintzberg (1982).

\section{Ajustement au poste de travail}

La deuxième configuration rassemble $43 \%$ des entreprises et $39 \%$ des salariés. Elle se caractérise par une politique de formation orientée vers l'ajustement au poste de travail à des fins de développement de l'entreprise. Ces organisations consacrent $2 \%$ de leur masse salariale en dépenses de formation s'adossant aux cours et stages comme mode quasi exclusif de formation. Si des opportunités de formation sont offertes, elles sont de courte durée et inégalement distribuées au sein des groupes socio-professionnels. De plus, les formes de participation ouvertes aux salariés et leurs latitudes d'action sont limitées par des entretiens le plus souvent réservés à une partie du personnel et une gouvernance de la formation impliquant peu leurs représentants. Cette caractéristique est confirmée par les salariés, dont près de $80 \%$ disent ne pas pouvoir modifier ou proposer une formation. Ces organisations se caractérisent aussi par une diffusion modeste des innovations organisationnelles. Le travail n'y est pas spécialement autonome, ne développe pas particulièrement de contenu cognitif, mais il n'est pas non plus particulièrement contraint. Le travail en équipe et la rotation des tâches y sont conformes à ce que l'on observe en moyenne. De leur côté, les salariés perçoivent le travail comme fondé sur une division en tâches simples et répétitives, qui rappelle le fonctionnement des organisations tayloriennes, sans en révéler toutefois toute l'ampleur des caractéristiques saillantes.

\section{Développement des compétences}

La troisième configuration, qui concerne $8 \%$ des entreprises et $21 \%$ des salariés, répond à une tendance lourde qui recherche une mobilisation plus grande de l'initiative des salariés. Les pratiques de travail en équipes autonomes, de rotation des tâches et surtout de management de la qualité y sont particulièrement diffusées. Concernant la politique de gestion des ressources humaines, on peut interpréter cette configuration 
comme développant plutôt une approche par les compétences qui favorise la performance de l'entreprise, la flexibilité et la polyvalence, le développement des normes qualités, la diffusion d'une culture commune. Les pratiques de formation plus généreuses sont rendues nécessaires par la plus grande autonomie attendue des salariés et la plus grande complexité des tâches. Avec une dépense de formation s'élevant à 4,6 \% de la masse salariale, les opportunités de formation sont importantes : on y déploie toutes sortes de formation, mais peu orientées vers des aspirations de promotions ou de mobilités horizontales. Cette configuration est également porteuse d'une gouvernance fondée sur une forte participation individuelle à des entretiens et sur une représentation par délégation plus élevée. En contrepoint, les salariés déclarent se voir imposer plus de contraintes (individuelles ou collectives) de rythme de travail et exercer des tâches répétitives. En termes d'organisation du travail, ce profil d'entreprise se rapproche du modèle type d'" autonomie contrôlée " que les employeurs développent pour concilier contrôle et mobilisation de leurs salariés au service de la performance de l'entreprise (Appay, 2005).

\section{Développement des capacités}

La quatrième configuration regroupe près d'un quart des salariés répartis dans $6 \%$ des entreprises du secteur privé et semi-public de dix personnes et plus. Selon les déclarations des entreprises, il existe une autonomie dans le travail, des pratiques de rotation des tâches, de résolution de problèmes et de management de la qualité, même si ces pratiques sont moins développées que dans les organisations de développement des compétences. En revanche, leur politique de formation ouvre l'accès à davantage d'opportunités : de formation, de mobilité professionnelle, éventuellement de promotion. La formation, l'information et la participation en sont les piliers. Ces organisations peuvent s'interpréter comme s'impliquant dans le développement professionnel de leurs salariés, faisant de la promotion des mobilités tant horizontales que verticales une dimension de la politique des ressources humaines. Les dépenses de formation atteignent $6,5 \%$ de la masse salariale, avec une généralisation des entretiens et des supports collectifs plus importants ici au regard du volume des représentants impliqués dans la gestion de la formation. Ainsi, ces organisations se caractérisent par une stratégie orientée à la fois vers le développement de l'entreprise et celui des salariés. Ainsi, avec Zimmermann (2014), nous pouvons avancer que ces organisations les plus capacitantes peuvent être définies comme des organisations apprenantes qui, de plus, encouragent et soutiennent les projets professionnels des salariés, comme en témoignent les déclarations des employeurs et des salariés. 
Tableau 1 - Le profil des quatre configurations d'entreprises

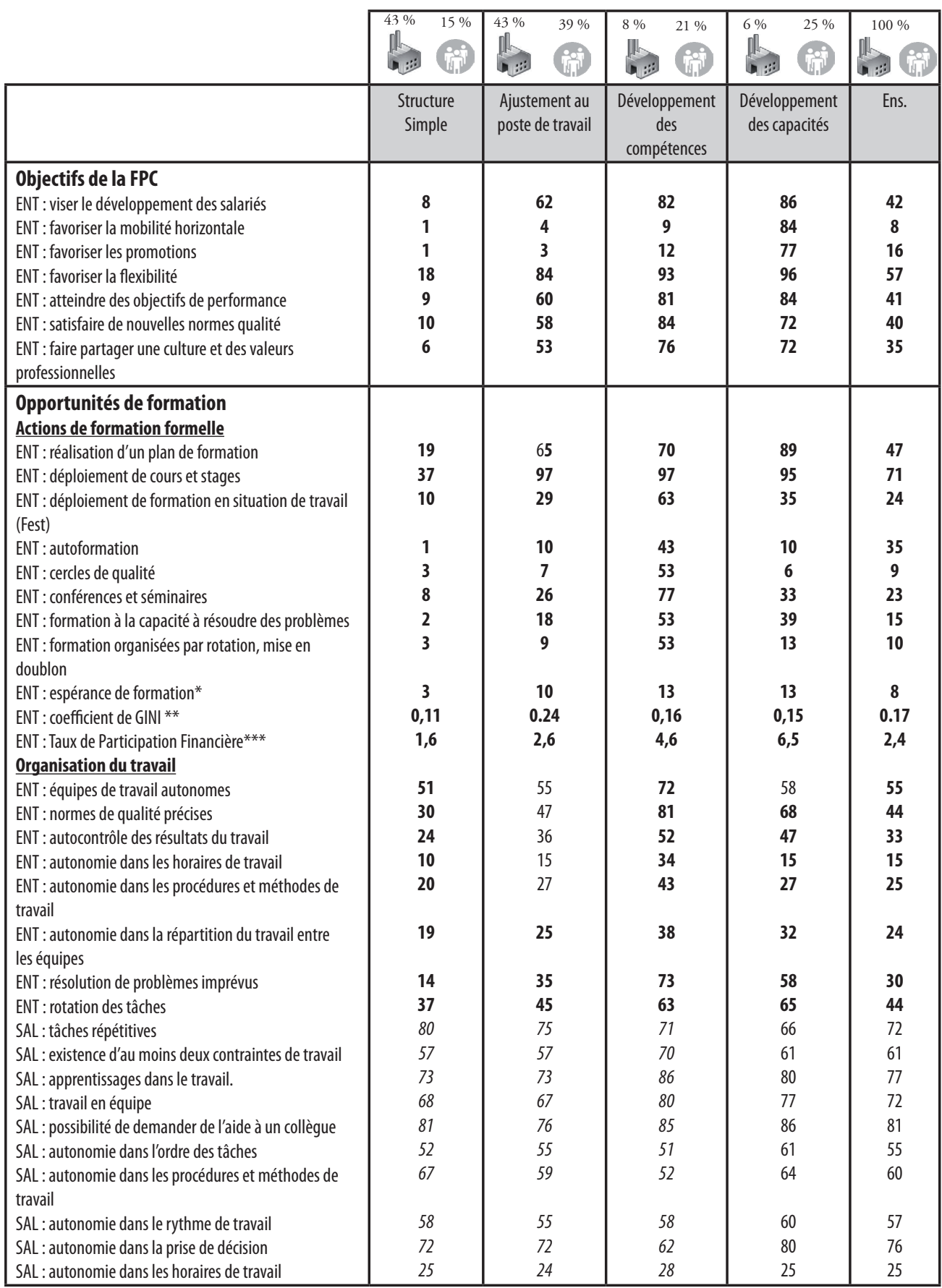




\begin{tabular}{|c|c|c|c|c|c|}
\hline \multicolumn{6}{|l|}{$\begin{array}{l}\text { Processus d'accès à la Formation } \\
\text { Modalités d'accès à la formation }\end{array}$} \\
\hline ENT : diffusion d'information sur la formation & 60 & 94 & 98 & 97 & 80 \\
\hline ENT : campagne d'information sur le DIF & 22 & 39 & 57 & 65 & 34 \\
\hline $\begin{array}{l}\text { ENT : entretiens professionnels auprès de tous les } \\
\text { salariés }\end{array}$ & 9 & 34 & 66 & 66 & 28 \\
\hline $\begin{array}{l}\text { ENT : entretiens professionnels de certains salariés } \\
\text { seulement }\end{array}$ & 17 & 34 & 24 & 23 & 25 \\
\hline ENT : implication des RP dans la gestion de la FPC & 13 & 33 & 43 & 61 & 27 \\
\hline $\begin{array}{l}\text { ENT : service ou personne responsable de l'organisation } \\
\text { de la FPC }\end{array}$ & 16 & 45 & 63 & 70 & 35 \\
\hline $\begin{array}{l}\text { SAL : Informé sur les possibilités de formation dans } \\
\text { l'entreprise }\end{array}$ & 33 & 62 & 80 & 82 & 66 \\
\hline $\begin{array}{l}\text { SAL: Pas de possibilité de modifier une proposition } \\
\text { de FPC }\end{array}$ & 93 & 9 & 18 & 17 & 12 \\
\hline Management & & & & & \\
\hline ENT : détermination d'objectifs au niveau collectif & 12 & 31 & 69 & 62 & 28 \\
\hline ENT: détermination d'objectifs au niveau individuel & 7 & 15 & 47 & 35 & 15 \\
\hline ENT: évaluation des besoins de compétences & 36 & 79 & 94 & 95 & 63 \\
\hline Réalisation & & & & & \\
\hline SAL : A suivi une formation au cours de l'année 2010 & 30 & 39 & 62 & 57 & 46 \\
\hline $\begin{array}{l}\text { SAL: A suivi une formation en lien avec un changement } \\
\text { dans l'emploi ou le travail }\end{array}$ & 20 & 22 & 41 & 38 & 29 \\
\hline $\begin{array}{l}\text { SAL : A suivi une formation qui a contribué ou contribue } \\
\text { à un projet professionnel }\end{array}$ & 11 & 12 & 16 & 20 & 15 \\
\hline
\end{tabular}

Légende:

ENT en gras : variables actives de quatre classes d'entreprises issues d'une classification hiérarchique ascendante sur l'enquête entreprise CVTS4.

SAL : en italique : variables illustratives issues du volet salariés : DIFES2 .

* : Espérance de formation : nombre d'heures moyen de formation par salarié.

** : Coefficient de GINI : degré d'inégalité de la distribution des salariés formés par CS par rapport aux effectifs de salariés par CS dans l'entreprise par la méthode de Silber et Deutsch.

Lecture : $80 \%$ des entreprises de la classe 4 déclarent viser le développement professionnel de leurs salariés à travers la formation professionnelle continue. $20 \%$ des salariés de la classe 4 déclarent avoir suivi une formation qui a contribué ou contribue à la réalisation d'un projet professionnel

Source : CVTS 4 et DIFES2. 


\section{L'organisation de développement des capacités en question(s)}

De rares entreprises mobilisent la formation à la fois pour le développement des objectifs de performance de l'entreprise et pour le développement des salariés et de leur évolution professionnelle. Cette double orientation caractérise les organisations de développement des capacités. Là où le développement des compétences se limite à l'acquisition de savoirs, le développement des capacités y ajoute une dimension déterminante : mieux articuler les formations à la réalisation des aspirations et projets professionnels. Cela ne se réduit pas à une norme de prise de responsabilité ou de carrière ascendante, mais comprend un ensemble de visées que chaque personne peut assigner à son travail, comme l'épanouissement et la réalisation de soi ou encore la reconnaissance sociale (Zimmermann, 2014).

\subsection{Quand se former coïncide avec une réalisation de valeur}

Nous voudrions introduire ici deux manières différentes, mais complémentaires, de concevoir la formation continue : d'une part, comme bien en soi, au sens d'objectif digne d'être poursuivi en tant que tel ; d'autre part, comme ouverture de possibles et potentiel de développement (Zimmermann, op. cit.). Au titre d'ouverture de possibles, comptent les réalisations de valeur, c'est-à-dire l'accomplissement de projets professionnels qu'autorise la formation. Ancrée dans le présent en tant que bien en soi, la formation est orientée vers le futur à travers les possibles qu'elle ouvre (cf. tableau 2).

Dans une perspective de capacités, la capacité à se former implique la possibilité, pour le travailleur, de poser des choix, de construire un projet professionnel en accord avec les besoins de l'organisation, mais encore avec la finalité qu'il attribue à la formation. Or, c'est dans les organisations de développement des capacités que les salariés s'avèrent être le plus en mesure de pouvoir engager une formation qui réponde à leurs propres aspirations. À certaines caractéristiques individuelles égales, à taille d'entreprise et secteur équivalents, les configurations jouent significativement sur la probabilité que la formation contribue à la réalisation d'un projet professionnel (cf. tableau 2). Si les salariés des configurations de développement des compétences ont plus de chances que les autres de suivre une formation, y compris en lien avec un changement dans l'emploi ou le travail, ce sont les salariés des configurations de développement des capacités qui s'avèrent être mieux placés pour réaliser une formation qui contribue à un projet professionnel. Ces statistiques reposent sur les déclarations des salariés. 
Tableau 2 - Configuration et réalisation d'une formation de valeur

\begin{tabular}{|l|c|c|c|c|}
\cline { 2 - 5 } \multicolumn{1}{c|}{} & \multicolumn{1}{c|}{$\begin{array}{c}\text { Structure } \\
\text { Simple }\end{array}$} & $\begin{array}{c}\text { Ajustement } \\
\text { au poste de } \\
\text { travail }\end{array}$ & $\begin{array}{c}\text { Développement } \\
\text { des compétences }\end{array}$ & $\begin{array}{c}\text { Développement des } \\
\text { capacités }\end{array}$ \\
\hline $\begin{array}{l}\text { Probabilité que les salariés déclarent } \\
\text { suivre une formation... }\end{array}$ & \multicolumn{3}{c|}{ Odd ratios } \\
\hline $\begin{array}{l}\ldots \text { au cours de l'année } 2010 \\
\ldots \text { en lien avec un changement dans } \\
\text { l'emploi ou le travail }\end{array}$ & $0,78^{* *}$ & Réf. & $\mathbf{1 , 9 0 ^ { * * * }}$ & $1.72^{* * *}$ \\
$\begin{array}{l}\ldots \text { qui a contribué ou contribue à un } \\
\text { projet professionnel }\end{array}$ & $0.98^{* * *}$ & Réf. & $1,72^{* * *}$ & $1,46^{* * *}$ \\
\hline
\end{tabular}

Le gras souligne, pour chaque ligne, la configuration la plus favorable.

Lecture : Ces résultats sont issus de modèles logistiques modélisant la probabilité de suivre trois registres de formation, en fonction des objectifs. Ils incluent l'âge, le diplôme, la catégorie socio-professionnelle, le genre des salariés, ainsi que le type de configuration, la taille, le secteur et l'appartenance à un groupe de l'entreprise. Les salariés d'une entreprise de type « développement des capacités » ont 1,39 fois plus de chances de réaliser une formation qui contribue à la réalisation d'un projet professionnel que ceux de la configuration « ajustement au poste de travail », tandis que par rapport à ces derniers, les salariés d'une entreprise de type « développement des compétences » n'ont pas plus de chances de réaliser une telle formation (1.01 fois plus de chances), pas plus que les organisations en Structure simple (pas de différence statistiquement significative).

Source : DIFES2.

Les entreprises les plus favorables au développement des capacités offrent des scénarios de flexibilité accordant une place centrale au développement des personnes au travail et dérogeant ainsi à la logique unilatérale de performance de l'entreprise. Elles s'avèrent aussi les plus aptes à favoriser la capacité des salariés à s'exprimer et à se faire entendre (cf. tableau 3). Toutes choses égales par ailleurs, ce sont en effet les salariés des organisations capacitantes qui déclarent le plus souvent être informés sur les possibilités de formation dans l'entreprise, discuter formation et carrière en entretien professionnel ou modifier les propositions de formation.

Si les écarts entre des configurations de développement des compétences et de développement des capacités peuvent paraître relativement faibles, des travaux de nature plus qualitative observent des relations significatives entre engagement participatif dans l'entreprise et organisation capacitante. Ainsi, pour Corteel et Zimmermann (2007, p. 35), dans les organisations les plus favorables au développement des capacités "la constitution du collectif s'appuie sur la participation et l'expression de la pluralité des opinions en présence ». D'autant plus que l'enquête Difes2 révèle d'importantes différences dans la faculté des salariés à se saisir des opportunités et des supports collectifs offerts par l'organisation (Subramanian et Zimmermann 2013 ; Zimmermann (2014). 
Tableau 3 - Probabilité des salariés de négocier sur la formation - liberté processus

\begin{tabular}{|l|c|c|c|c|}
\cline { 2 - 5 } \multicolumn{1}{c|}{} & \multicolumn{1}{c|}{$\begin{array}{c}\text { Structure } \\
\text { Simple }\end{array}$} & $\begin{array}{c}\text { Ajustement au } \\
\text { poste de travail }\end{array}$ & $\begin{array}{c}\text { Développement } \\
\text { des compétences }\end{array}$ & $\begin{array}{c}\text { Développement } \\
\text { des capacités }\end{array}$ \\
\hline $\begin{array}{l}\text { Probabilité que les salariés } \\
\text { déclarent... }\end{array}$ & \multicolumn{3}{|c|}{ Odd ratios } \\
\hline $\begin{array}{l}\ldots \text { être informés sur les possibilités } \\
\text { de formation dans l'entreprise } \\
\ldots \text { discuter formation et carrière } \\
\text { dans le cadre de l'entretien } \\
\text { professionnel } \\
\ldots \text { modifier les propositions de } \\
\text { formation dans l'entreprise }\end{array}$ & $0.78^{* *}$ & Réf. & $1,73^{* * *}$ & $1,9^{* * *}$ \\
\hline
\end{tabular}

Le gras souligne, pour chaque ligne, la configuration la plus favorable.

Lecture: Ces résultats sont issus de modèles logistiques modélisant la probabilité de suivre trois registres de formation. Ils incluent l'âge, le diplôme, la catégorie socio-professionnelle, le genre des salariés, ainsi que le type de configuration, la taille, le secteur et l'appartenance à un groupe de l'entreprise. Les salariés sortant d'une entreprise de type « développement des capacités » ont 1,96 fois plus de chances de modifier une proposition de formation que ceux de la configuration « ajustement au poste de travail », tandis que par rapport à ces derniers, les salariés d'une entreprise de type « développement des compétences » ont 1,70 fois plus de chances de modifier une telle proposition de formation (1.01 fois plus de chances), et que les organisations en Structure simple n'ont pas plus de chances (pas de différence statistiquement significative).

Source : DIFES2.

\subsection{Le développement des capacités : une affaire de taille, de secteur ou de groupe socio-professionnel ?}

Si les entreprises de grande ou moyenne taille semblent se prêter à une politique de capacités, la taille ne semble pas avoir un caractère discriminant : plus de la moitié des entreprises capacitantes ont des effectifs inférieurs à 50 salariés. Une grande taille est corrélée avec l'existence d'opportunités importantes, mais à l'inverse, une taille moindre ne prive pas d'opportunités et encore moins de pouvoir d'action (cf. tableau 2). À l'exception des entreprises en "structure simple ", qui se distinguent par leur petite taille, la taille imprime faiblement sa marque sur les configurations d'entreprise. Par ailleurs, si les organisations capacitantes sont plus développées dans l'industrie et les activités immobilières, on les retrouve dans tous les secteurs. En outre, les cadres et les professions intermédiaires sont plus nombreux dans les organisations de développement des compétences et de capacités. Pour autant, comme dans l'ensemble des entreprises, les deux organisations emploient majoritairement des employés et des ouvriers. Au final, on n'observe pas de réel déterminisme en matière de politique de formation qui s'expliquerait par la taille, le secteur ou la composition socio-professionnelle de l'entreprise. 
Tableau 4 - Les caractéristiques structurelles des quatre configurations d'entreprises

(en pourcentage des entreprises)

\begin{tabular}{|c|c|c|c|c|c|}
\hline & $\begin{array}{l}\text { Structure } \\
\text { Simple }\end{array}$ & $\begin{array}{l}\text { Ajustement } \\
\text { au poste de } \\
\text { travail }\end{array}$ & $\begin{array}{l}\text { Développement } \\
\text { des compétences }\end{array}$ & $\begin{array}{l}\text { Développement } \\
\text { des capacités }\end{array}$ & Ens. \\
\hline $\begin{array}{l}\text { Taille des Entreprises } \\
\text { Entreprises de } 10 \text { à } 49 \text { salariés } \\
\text { Entreprises de } 49 \text { à } 499 \text { salariés } \\
\text { Entreprises de plus de } 500 \\
\text { salariés }\end{array}$ & $\begin{array}{l}94 \\
6 \\
0\end{array}$ & $\begin{array}{c}80 \\
19 \\
1\end{array}$ & $\begin{array}{c}69 \\
26 \\
5\end{array}$ & $\begin{array}{c}56\left(^{*}\right) \\
35 \\
9\end{array}$ & $\begin{array}{c}84 \\
15 \\
1\end{array}$ \\
\hline $\begin{array}{l}\text { Secteur d'activité } \\
1 \text { Industrie } \\
2 \text { Construction et bâtiments, } \\
\text { génie civil, travaux de construc- } \\
\text { tion spécialisés } \\
3 \text { Commerce et réparation } \\
\text { automobile, commerce de gros } \\
\text { et de détail } \\
4 \text { Transports terrestres, par eau } \\
\text { et aérien ; services auxiliaires des } \\
\text { transports, activité de poste et } \\
\text { de courrier } \\
5 \text { Hébergement, restauration } \\
6 \text { Édition ; production de films } \\
\text { cinématographiques ; édition } \\
\text { musicale ; télécommunications } \\
7 \text { Activités des services finan- } \\
\text { ciers, assurance ; activités } \\
\text { auxiliaires de services financiers } \\
\text { et d'assurance } \\
8 \text { Activités immobilières }\end{array}$ & $\begin{array}{l}22 \\
8\end{array}$ & $\begin{array}{l}7 \\
4 \\
3 \\
\\
26\end{array}$ & $\begin{array}{l}7 \\
5 \\
2 \\
39\end{array}$ & $\begin{array}{l}7 \\
7 \\
6 \\
29\end{array}$ & $\begin{array}{l}19 \\
15\end{array}$ \\
\hline $\begin{array}{l}\text { Groupes socio-professionnels } \\
\text { Cadres } \\
\text { Professions intermédiaires } \\
\text { Employés } \\
\text { Ouvriers }\end{array}$ & $\begin{array}{c}9 \\
11 \\
42 \\
38\end{array}$ & $\begin{array}{l}12 \\
19 \\
28 \\
41\end{array}$ & $\begin{array}{l}23 \\
24 \\
22 \\
31\end{array}$ & $\begin{array}{l}24 \\
22 \\
27 \\
27\end{array}$ & $\begin{array}{l}17 \\
20 \\
28 \\
35\end{array}$ \\
\hline
\end{tabular}

Lecture : $\left(^{*}\right) 56 \%$ des entreprises de développement des capacités ont une taille comprise entre 10 et 49 salariés.

Source : Enquête CVTS4.

\section{Conclusion}

La recherche présentée dans cet article met en évidence et analyse la diversité des politiques de formation des entreprises françaises. À ce titre, elle montre que les organisations de "développement des compétences » ne constituent pas la seule voie possible de développement des dynamiques de formation dans le travail.

En fait, deux configurations y concourent : celle de "développement des compétences » et celle de « développement des capacités ». Mais ce n'est que dans cette dernière qu'existe la possibilité réelle, pour tous les travailleurs, d'exprimer et de faire entendre leur point de vue, de poser des choix en matière de formation et de construire un projet professionnel 
en accord avec les besoins de l'organisation, mais aussi avec la finalité que chacun attribue à la formation. Ainsi, les organisations capacitantes se révèlent être les seules configurations susceptibles d'offrir des opportunités et des mécanismes structurants mettant les salariés en capacité de se former et d'avoir une prise sur leur devenir professionnel. Elles représentent aujourd'hui près d'un quart des salariés, répartis dans $6 \%$ des entreprises du secteur privé et semi-public de dix personnes et plus.

Si les réformes de la formation continue, depuis près de quinze ans, valorisent l'initiative individuelle et dotent progressivement les personnes de moyens accrus susceptibles de soutenir leur capacité d'action, il reste que celle-ci ne dépend pas du seul volontarisme institutionnel ou individuel, mais est fortement tributaire des politiques et des pratiques des entreprises. À la veille de la réforme de 2014, seulement $6 \%$ des entreprises avaient mis en ouvre les conditions susceptibles de garantir des ressources et opportunités de valeur, c'est-à-dire construites au cours d'une interaction dialectique individu-organisation par l'exercice de la démocratie. De ce point de vue, les procédures permettant aux salariés d'exprimer et de faire entendre leur voix en matière de formation et d'évolution professionnelle se révèlent être un maillon central.

L'enjeu consiste à élargir le cercle de ces entreprises capacitantes. La loi de 2014 est la première en matière de formation à se confronter autant à la question de l'effectivité des droits à une formation qualifiante (Caillaud, 2014) et de la capacité à se former des salariés, au-delà de l'énonciation de droits formels. Dans cette perspective, les partenaires se sont accordés pour aménager des espaces d'expression individuelle (entretiens professionnels obligatoires tous les deux ans), en dotant les salariés de possibilités de recours pour faire valoir leurs droits en matière de formation et d'évolution professionnelle (abondement du compte personnel en cas de défaillance de l'entreprise). L'objectif d'égale capacité à se former et à maîtriser son destin professionnel, au sens de liberté positive soutenue par des moyens d'agir, devient l'innovation majeure et un pari sur l'avenir.

La loi travail s'inscrit dans la continuité de la réforme de la formation professionnelle introduite par la loi du 5 mars 2014. Cependant, elle assouplit la notion d'action de formation et ouvre à une diversité de modalités d'apprentissage auxquelles l'entreprise peut recourir dès lors qu'elle finance la formation sur ses fonds propres (Luttringer, 2016).

L'apprentissage en situation de travail devient dès lors un domaine à part entière à enquêter. À cet égard, de récents travaux montrent qu'une approche des apprentissages à travers les dynamiques de travail des salariés constitue un levier à actionner afin que tous, y compris et peut-être surtout les moins qualifiés, puissent trouver les moyens d'évoluer professionnellement (Fournier et al., 2017). Mais seule l'enquête empirique, en confrontant cette nouvelle approche à sa mise en œuvre dans les entreprises, permettra d'en apprécier l'impact et les effets sur la capacité/incapacité des salariés à infléchir leur parcours.

Au travers des quatre dimensions des capacités - les objectifs de la formation, la libertéopportunités, la liberté-processus et les réalisations de valeur - l'approche par les capacités 
ouvre la voie à des approfondissements empiriques qui permettront de mieux spécifier la liberté des personnes de construire leur devenir à travers les diverses formes d'apprentissage dans l'entreprise. Par là même, nous semble-t-il, on sera aussi mieux outillé pour éclairer les débats entre les partisans d'une vision très optimiste des récentes réformes et ses détracteurs, et apporter une contribution significative à l'évaluation de l'objectif de sécurisation des parcours par la formation tout au long de la vie.

\section{Bibliographie}

Accord national interprofessionnel du 20 septembre 2003 relatif à « l'accès des salaries à la formation tout au long de la vie professionnelle".

Appay B. (2005), La dictature du succès, Le paradoxe de l'autonomie contrôlée et de la précarisation, Paris, L'Harmattan.

Bonvin J.-M (2005), « La démocratie dans l'approche d'Amartya Sen ", L'Économie politique $3 / 2005, n^{\circ} 27$, pp. 24-37

Bonvin J.-M., Farvaque N. (2008), Amartya Sen. Une politique de la liberté, Paris, Michalon, $128 \mathrm{p}$.

Bonvin J.-M. \& Orton M. (éds) (2009), "Activation policies and organisational innovation in the capability, perspective", International Journal of Sociology and Social Policy, December, vol. 29, issue 11-12.

Boyer R., Durand J.-P. (1993), L'après fordisme, Paris, Syros.

Caillaud P. (2014), "Un droit à la qualification enfin effectif ?", Droit Social, n 22, pp. 1000-1006.

Caillaud P. (2016), "Les avatars de la notion de compte en droit du travail », Droit Social, $\mathrm{n}^{\circ} 10$, octobre, pp. 8006-8011.

Caillaud P., Zimmerman B. (2011), «Sécurisation des parcours et liberté professionnelle : de la "flexicurité " aux capacités ", Formation Emploi, n¹13, pp. 33-48

Coenen-Huther J. (2007), "Classifications, typologies et rapport aux valeurs », Revue européenne des sciences sociales, XLV-138.

Commission européenne (2007), Vers des principes communs de flexicurité : des emplois plus nombreux et de meilleure qualité en combinant flexibilité et sécurité, Bruxelles, Com 359 final.

Corteel D., Zimmermann B. (2007), "Capacités et développement professionnel ", Formation Emploi, $\mathrm{n}^{\circ}$ 98, pp. 25-39.

Dayan J.-L. (2016), "Comment donner corps à la sécurité professionnelle? », Droit social, $\mathrm{n}^{\circ} 10$, octobre, pp. 840-847. 
De Munck J., Zimmermann B. (2008), La liberté au prisme des capacités. Amartya Sen audelà du libéralisme, Raisons Pratiques, Editions de l'EHESS, 333 p.

De Gaulejac V. (2005), La société malade de la gestion, Paris, Seuil.

De Gaulejac V., Hanique F. (2015), Le capitalisme paradoxant, Paris, Seuil.

Durand J.P, (2004), La chaîne invisible. Travailler aujourd'hui : flux tendu et servitude volontaire, Paris, Seuil.

Elster J. (1983), Sour Grapes, Cambridge University Press, Cambridge.

Etzioni A. (1971), Les organisations modernes, Editions Duculot.

Farvaque N. (2008), « Faire surgir des faits utilisables. Comment opérationnaliser l'approche par les capacités ? " in De Munck J., Zimmermann B, La liberté au prisme des capacités : Amartya Sen au delà du libéralisme, Raisons pratiques, ${ }^{\circ} 18, \mathrm{pp}$.51-80 .

Formation Emploi (2007), " Pour une approche par les capacités », numéro spécial 98, avril-juin.

Fournier C., Lambert M., Marion I. (2017), « Le travail au coeur des apprentissages en entreprise ", Bref-Céreq, n 353, avril, 4 p.

Giauque D. (2003), La bureaucratie libérale, Paris, L'Harmattan.

Giddens A. (1998), The third way, Cambridge, Polity Press.

Lambert M., Vero J. (2013), “The capability to aspire for continuing training in France The role of the environment shaped by corporate training policy", International Journal of Manpower, Vol. 3, n 4, , pp. 305-325.

Lambert M., Vero J., Zimmermann B. (2012),"Vocational Training and professional development", International Journal of Training and Development, Vol. 16/3, September, pp. 164-182.

Livian Y.-F. (2008), Organisation. Théories et Pratiques, Paris, Dunod.

Loi $\mathrm{n}^{\circ} 71-575$ du 16 juillet 1971 portant organisation de la formation professionnelle continue dans le cadre de l'éducation permanente.

Loi n²004-391 du 4 mai 2004 relative à la formation professionnelle tout au long de la vie et au dialogue social.

Loi n ${ }^{\circ}$ 2014-288 du 5 mars 2014 relative à la formation professionnelle, à l'emploi et à la démocratie sociale.

Loi n ${ }^{\circ}$ 2016-1088 du 8 août 2016 relative au travail, à la modernisation du dialogue social et à la sécurisation des parcours professionnels.

Lorenz E., Valeyre A. (2005), "Les formes d'organisation du travail dans les pays de l’Union européenne ", Travail et Emploi, n 102, avril, pp. 91-105. 
Luttringer J.-M. (2013), «L'ANI du 14 décembre 2013 : nouvelles dynamiques et grandes fragilités juridiques ", Chronique, $\mathrm{n}^{\circ} 82$, décembre.

Luttringer J.-M. (2016), " La réforme de la formation professionnelle deux ans après.1 Questions évaluatives et préconisations ", Chronique, n 104, février.

Luttringer J.-M. (2016), « Le pouvoir sur la formation dans l'entreprise après la loi travail ", Chronique, $\mathrm{n}^{\circ} 113$, octobre.

Merle V., Lichtenberger Y. (2001) «Formation et éducation tout au long de la vie, 19712001 : deux réformes, un même défi ", Formation Emploi, n 76.

Mintzberg H. (1982), Structure et dynamique des organisations, Paris, Éditions d'Organisation.

Mintzberg (1986), Le pouvoir des organisations, Paris, Éditions d'Organisation.

Nizet J., Pichault F. (2001), Introduction à la théorie des configurations. Du "one best way» à la diversité organisationnelle, Coll. " Management ", De Boeck Université, Bruxelles.

Perrow C. (1970), Organizational Analysis: A Sociological View, Tavistock Press.

Pichault F., Nizet J. (2013), Les pratiques de gestion des ressources humaines. Conventions, contextes et jeux d'acteurs, coll. « Points/Économie ", Paris, Éditions du Seuil.

Salais R. (2007), «Introduction : À la recherche de principes novateurs pour l'action publique ", Formation Emploi, n ${ }^{\circ}$ 98, pp. 5-8.

Schmidt G., B. Gazier (eds.) (2002), The Dynamics of Full Employment: Social Integration Through Transitional Labour Markets. Cheltenham, UK and Brookfield, US: Edward Elgar.

Sen A.-K. (1985), Commodities and Capabilities, Amsterdam, North Holland.

Sen A.-K. (1992), Inequality Reexamined, Oxford, Oxford University Press.

Sen A. K. (1999), Éthique et économie, Paris, PUF, 364 p.

Sen A. K. (2000), Un nouveau modèle économique. Développement, justice et liberté, Paris, Odile Jacob.

Sen A.K. (2005), Rationalité et liberté en économie, Paris, Odile Jacob.

Sen A. K (2006), La démocratie des autres: pourquoi la liberté n'est pas une invention de l'Occident, Paris, Rivages Poche.

Sen A.-K. (2009), Lidée de justice, Paris, Flammarion.

Senge P. (2000), La cinquième discipline, Paris, First Éditions.

Sigot J.-C, Vero J. (2014), "Politiques d'entreprise et sécurisation des parcours : un lien à explorer ", Bref-Céreq, n³18, janvier. 
Subramanian D., Zimmermann B. (2013), “Training and Capabilities in French Firms: How Work and Organisational Governance Matter", International Journal of Manpower, Vol. 34, ${ }^{\circ}$ 4, pp. 326-344.

Supiot A. (dir.) (1999), Au-delà de l'emploi. Transformations du travail et devenir du droit du travail en Europe, Paris, Flammarion, 321 p.

Verd J.-M. et Vero J. (2011) «Pourquoi la flexicurité mérite-t-elle qu’on en débatte à partir de l'approche par les capacités ? ", Introduction au Dossier « La flexicurité à l'aune de l'approche par les capacités ", Formation Emploi, n 113, janvier-mars.

Vero J., Bonvin J.-M., Lambert M. \& Moachon E. (2012), “Decoding the European dynamic employment security indicator through the lens of the capability approach. A comparison of the United Kingdom and Sweden", Transfer, Vol. 18, n 1, pp. 55-67.

Womack J. P., Jones D. T., Roos D. (1990), The Machine that changed the World, Rawson Associates, New York.

Zimmermann B (2014), "Ce que travailler veut dire. Sociologie des capacités et des parcours professionnels ", Paris, Economica (Études sociologiques), (ed. originale 2011), 233 p. 


\section{L'ORIENTATION SCOLAIRE ET PROFESSIONNELLE}

Revue de l'Institut national d'étude du travail et d'orientation professionnelle

Numéro 46/1 (mars 2017)

Numéro thématique - Lyda Lannegrand-Willems \& Emmanuelle Vignoli (Coord.) Adolescence et orientation (Volume 1)

Lyda Lannegrand-Willems \& Emmanuelle Vignoli Introduction

Henri Lehalle

Les changements idéologiques à la période de l'adolescence : construction, choix, ruptures

Fréderic Fourchard, Marie Huet-Gueye, Ania Beaumatin, Véronique Rouyer, Hervé Larroze-Marracq, Yoan Mieyaa, \& Lyda Lannegrand-Willems

Citoyenneté et orientation à l'adolescence : dialectique des processus identitaires et de socialisation

Catherine Loisy \& Émilie Carosin

Concevoir et accompagner le développement du pouvoir d'agir des adolescentes et des adolescents dans leur orientation

Poyraz Sahin \& Isabelle Olry-Louis

La formation des intentions professionnelles des adolescent.e.s se reconnaissant dans une orientation sexuelle minoritaire

Fernando Núñez-Regueiro

Le décrochage scolaire comme processus de stress : les adolescent.e.s à l'épreuve des croyances de justice et d'auto-efficacité

Abonnement 2017 (4 numéros par an + un HS)

France : 112 Euros, Étranger : 118 Euros

OSP - Service abonnement

41 rue Gay-Lussac - 75005 Paris

0144107833 - inetop-osp@cnam.fr 